\title{
FIELD ANALYSIS OF AN INDUCTION COOKER WITH SQUARE 9-COIL SYSTEM BY APPLYING DIVERSE EXCITING PATTERNS
}

\author{
L.C. Meng*, K.W.E. Cheng*, P.C.K.Luk ${ }^{\dagger}$ \\ *Department of Electrical Engineering, The Hong Kong Polytechinic University, Hong Kong, \\ eeecheng@polyu.edu.hk \\ + School of Engineering, Cranfield University, U.K. \\ p.c.k.luk@cranfield.ac.uk
}

Keywords: Coils, electromagnetic analysis, eddy currents, finite element method, induction heating.

\begin{abstract}
This paper proposes a novel square 9-coil $(3 \times 3)$ matrix system for induction cooking applications in order to improve the heating performance. By means of finite element method (FEM) analysis, three different exciting modes for the proposed system are thoroughly investigated. The magnetic field and eddy currents distribution are studied for each case. Furthermore, thermal field computation is conducted to analyze and compare the heating performance for the proposed system with different exciting modes. The thermal field computation results indicate that the proposed square 9coil system can effectively and significantly enhance the heating performance in terms of better uniformity and heating efficiency over conventional single coil systems.
\end{abstract}

\section{Introduction}

Nowadays, induction cooking is becoming a viable alternative to conventional cooking in the food and catering industry. It is more efficient than cooking with heating filaments, and at least 3 times more efficient than that of gas heating. It also provides a quiet, safe and low radiation cooking environment. However, the traditional single coil induction cooker generally suffers from the problems of localized thermal distribution and the resulting fast "aging" of the induction pan [1]. The geometry and the excitation of the induction coils fundamentally determine the heating area and thermal distribution of an induction cooker. Whilst replacing a single coil with smaller multiple coils could improve the distribution of magnetic field as well as the heat pattern of the induction cooker, there are further complications arising from the magnetic interference and electric insulation among the coils. Thus, comprehensive analyses on the magnetic and thermal fields are crucial for the optimized design of induction cookers with multi-coils.

This paper proposes a novel induction cooker with 9 square coils arranged as a $3 \times 3$ matrix, which not only addresses the problem of uneven thermal distribution, but also improves the heating performance. Three simply modes of excitation are proposed and implemented in the 9-coil system by means of Finite Element Method (FEM) models. The eddy currents and power losses distribution on the induction pan by the three exciting modes are investigated and compared using the predicted results of the FEM models. Furthermore, the thermal rising-up processes for the proposed induction cooker are also studied in order to assess the effectiveness of the heating. The FEM results provide insightful information and understanding for the design and optimization of the induction cooker with a square 9-coil system.

\section{Heating source distribution analysis}

The proposed system is a $3 \times 3$ square matrix configuration, consisting of 9 identical squared wound coils as depicted in Fig.1(a). This coil arrangement is relatively simple and compact, and provides maximum heating area per unit pan area. The FEM model of the coil system is shown in Fig.1(b), where the details of the parameters are given in Table 1. The applied driving current for each coil is chosen to be $10 \mathrm{~A}$ at $20 \mathrm{kHz}$ for this base-line study, which is a typical specification for induction heating. Since the relative phase differences among the exciting currents significantly influence the magnetic flux and eddy currents distribution due to proximity effects among the distributed coils [3], three different exciting modes with varying phase differences are investigated for the evaluation and optimization of the heating source distribution.
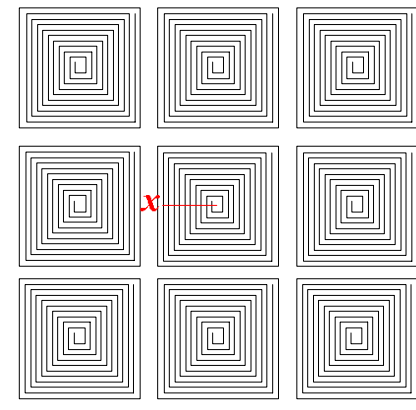

(a)



(b)
Fig. 1: Proposed square 9-coil system (a) Coils arrangement illustration, (b) FEM model 


\begin{tabular}{cc}
\hline \multicolumn{2}{c}{ Coils } \\
\hline Relative permeability & 1 \\
Electrical conductivity & $5.8 \mathrm{e} 7 \mathrm{~S} / \mathrm{m}$ \\
Total surface side length & $0.16 \mathrm{~m}$ \\
Length x (each) & $0.05 \mathrm{~m}$ \\
Distance between coils & $0.01 \mathrm{~m}$ \\
Number of turns (each) & 14 \\
\hline \multicolumn{2}{c}{ Induction pan } \\
\hline Relative permeability & 1500 \\
Electrical conductivity & $1 \mathrm{e} 6 \mathrm{~S} / \mathrm{m}$ \\
Thickness & $0.002 \mathrm{~m}$ \\
Radius & $0.25 \mathrm{~m}$ \\
\hline \multicolumn{1}{c}{ Insulator } \\
\hline Relative permeability & 1 \\
Thickness & $0.007 \mathrm{~m}$ \\
\hline
\end{tabular}

Table 1: Parameters for the FEM model

\subsection{In-phase exciting mode}

The first exciting mode under study is the most obvious one, with all the 9 coils being excited in phase. The details of the distributions of the resultant eddy currents $J$ are shown in Fig. 2. Since the coils are excited by in- phase driving currents, with the ' + ' sign denoting current flowing anti-clockwise on the plane as shown in Fig.2(a), the induced eddy current density at the boundaries of all coils with adjacent coils is reduced due to magnetic field cancellation as shown in Fig.2(b). These areas with low eddy current densities are located in the inner and central parts of the cooking pan, with 5 troughs of low magnetic flux which significantly affect the efficiency of cooking due to uneven thermal distribution. It is also noted that the peaks magnetic flux, at 0.56T, are around the outer boundaries of the pan, rather than at the central area where the peak heating effect is preferred.

\section{2 $120^{\circ}$ Out of phase exciting mode}

From the above analysis, it is known that in-phase exciting mode is not a preferred way to optimize eddy current distribution. A simple $180^{\circ}$ out of phase exciting pattern, as depicted in Fig.3(a), is used to improve heating performance. The resultant eddy currents $J$ distribution is shown in Fig.3(b). The lagging in phase of the exciting currents results in adjacent coils aiding each other in the boundaries of the inner coils, in particular in the central parts of the pan. The peak of the magnetic field is about $0.62 \mathrm{~T}$, which is $7 \%$ higher than that of the in-phase excitation.

Compared to the eddy currents distribution in Fig.2(b), the results obtained in Fig.3(b) is markedly improved. There are less low eddy currents areas in the boundaries among coils with more uniform distribution, with only 3 troughs, 2 fewer than that of the in-phase excitation. Instead of magnetic field cancellation, the boundary areas between adjacent coils have enhanced magnetic field due to out of phase driving currents.

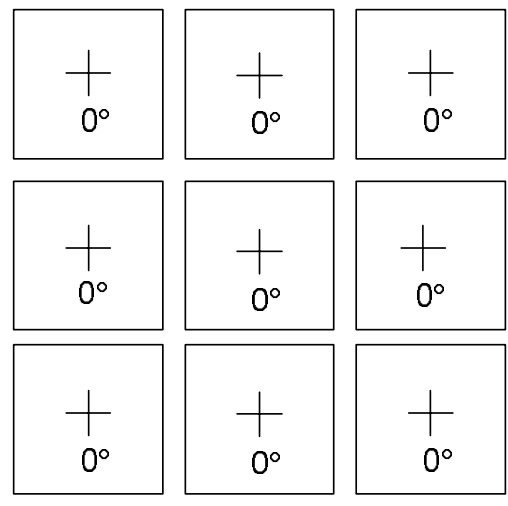

(a)



(b)

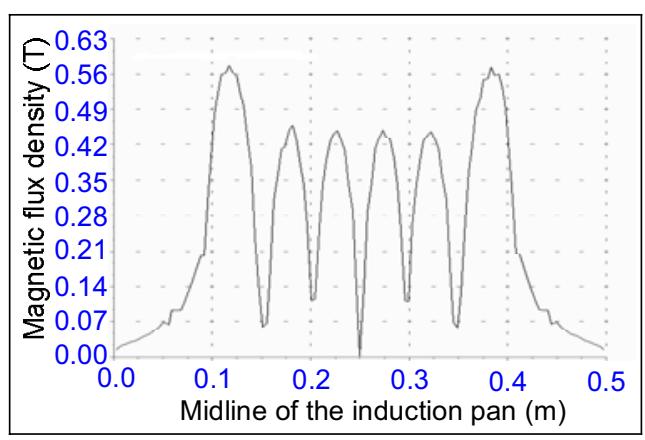

(c)

Fig. 2: Excited by in phase driving currents, (a) exciting pattern illustration, (b) resultant eddy currents distribution, (c) magnetic field distribution along the midline of the pan.

However, it is noted that lower magnetic field and eddy currents appear in the areas between the diagonal coils. This is because that the coil in the centre is not driven out of phase to all the coils around it.

\section{$2.3120^{\circ}$ phase shift exciting mode}

In order to further improve the eddy currents distribution on the pan, the driving currents are $120^{\circ}$ phase shifted among the 

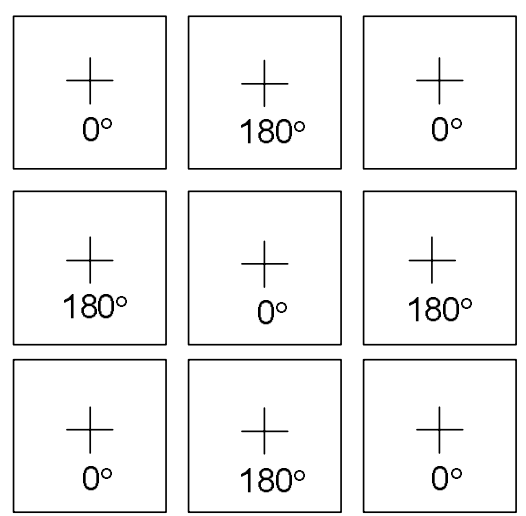

(a)



(b)

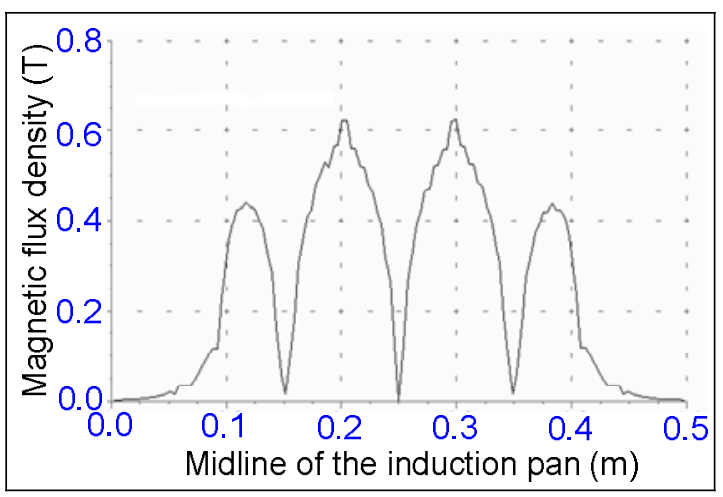

(c)

Fig. 3: Excited by out of phase driving currents, (a) exciting pattern illustration, (b) resultant eddy currents distribution, (c) magnetic field distribution along the midline of the pan.

coils. The eddy currents distribution obtained by FEM analysis is shown in Fig. 4.

\section{Thermal field analysis}

Power losses as the heating source $Q_{e}$ can be calculated based on the eddy currents distribution results as:

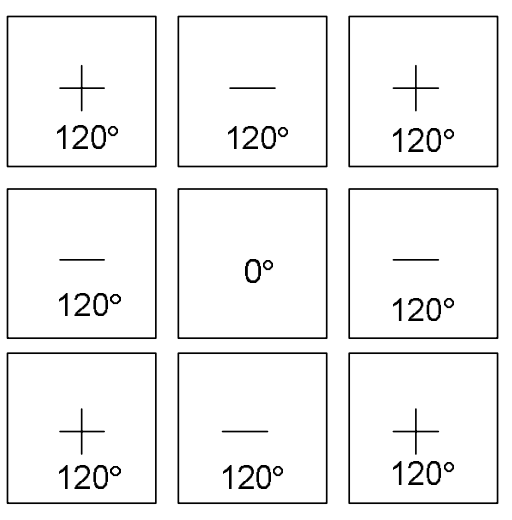

(a)

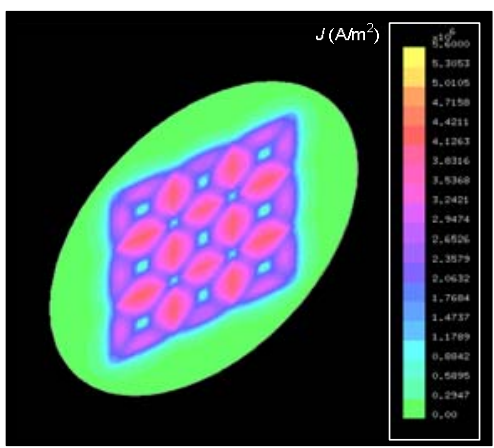

(b)

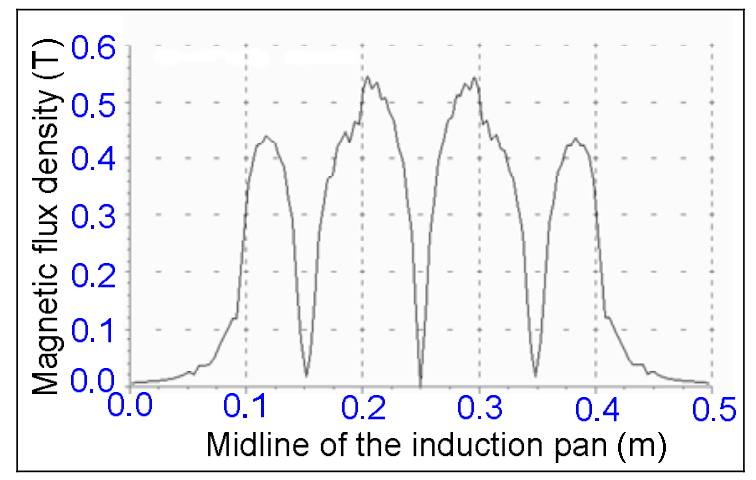

(c)

Fig. 4: Excited by $120^{\circ}$ phase shifted driving currents, (a) exciting pattern illustration, (b) resultant eddy currents distribution, (c) magnetic field distribution along the midline of the pan.

$$
Q_{e}=\frac{|J|^{2}}{\sigma}
$$

where $\sigma$ denotes the electrical conductivity of the induction pan. 
On the basis of the obtained heating source $Q_{e}$, the Transient Diffusion Equation in (2) is therefore applied for the thermal field calculation,

$$
\nabla \cdot(\lambda \nabla T)-c \rho \frac{\partial T}{\partial t}=-Q_{e}
$$

where $\lambda$ denotes thermal conductivity, $c$ stands for specific heat, and $\rho$ represents mass density. For induction cooking applications, the temperatures on the pan is usually far below the Curie temperature of the pan material [2]. Hence the thermal properties of the pan generally have linear relationships with temperature within this range. The heating source therefore directly influences the obtained temperature.

The thermal field on the induction pan with the proposed square 9-coil system is computed based on (1) and (2) by defining boundary conditions. The temperature on the midline of the pan, as indicated in Fig.1(b), when applying the in phase exciting mode is given in Fig. 5. It can be observed that

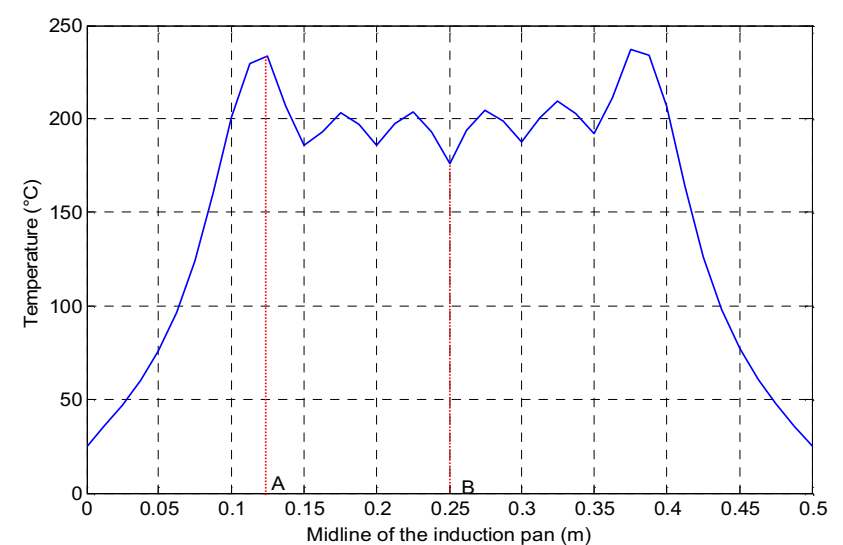

(a)

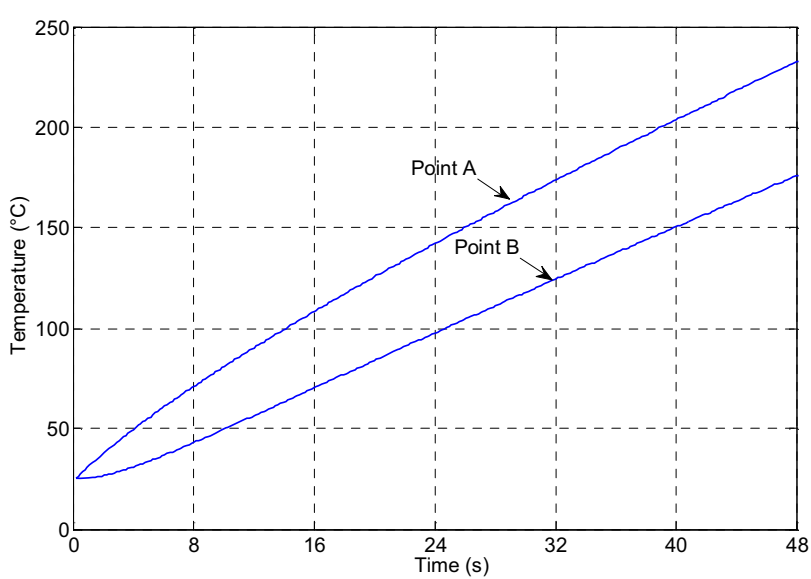

(b)

Fig. 5: Thermal field computation results for in phase exciting mode, (a) temperature distribution on the midline of the pan, (b) temperature rise profile. there are six temperature peaks, where the two highest peaks are at $230^{\circ} \mathrm{C}$ at either end of the midline, and the four lower peaks are around $200^{\circ} \mathrm{C}$ lying in between. The lowest temperature within the bulk of the cooking area is at the mid point at around $175^{\circ} \mathrm{C}$. Comparing to traditional single coil induction cooker, its thermal distribution is more uniform. The highest and lowest temperature points along the entire midline are indicated in Fig.5(a) as points " $A$ " and point "B", with a temperature $55^{\circ} \mathrm{C}$. Their rising processes are plotted in Fig.5(b). Within around 50 seconds, the highest and lowest temperatures on the midline of the induction pan are settling at $230{ }^{\circ} \mathrm{C}$ and $175^{\circ} \mathrm{C}$ respectively.

It is envisaged that the heating performance for the $180^{\circ}$ outof-phase excitation will be similar to that of the $120^{\circ}$ phase shifted excitation, only the latter will be investigated. The temperature on the midline of the pan as indicated in Fig.1(b) when applying the $120^{\circ}$ phase shifted exciting mode is given in Fig.6. It can be observed that there are four temperature peaks, with the two lower peaks at $200^{\circ} \mathrm{C}$ at the either end of

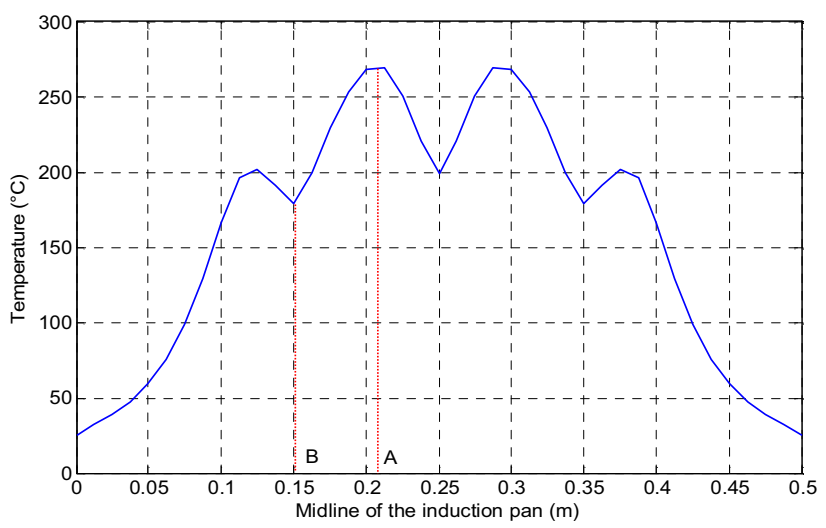

(a)

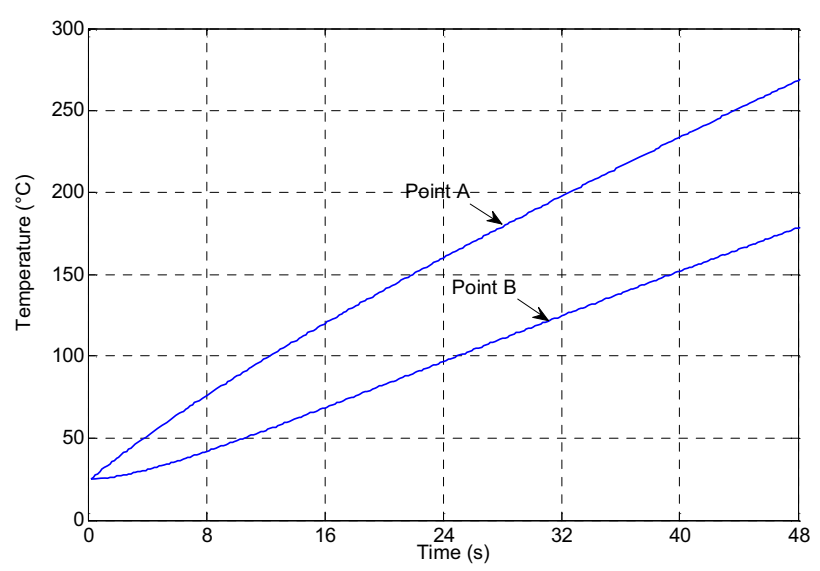

(b)

Fig. 6: Thermal field computation results for $120^{\circ}$ phase shifted exciting mode, (a) temperature distribution on the midline of the pan, (b) temperature rise profile. 
the midline, and two higher peaks at $270^{\circ} \mathrm{C}$ in between. The highest and lowest temperature points are indicated in Fig.6(a) as points " $A$ " and point " $B$ ". The lowest temperature at point $\mathrm{B}$ is $180^{\circ} \mathrm{C}$ within the bulk of the cooking pan area, and the temperature difference between the maximum and minimum is around $90^{\circ} \mathrm{C}$. Thus, it can be argued that the thermal distribution in Fig.6(a) is less uniform than that of Fig.5(a). The temperature rising processes for points $\mathrm{A}$ and $\mathrm{B}$ are plotted in Fig.6(b). Within around 50 seconds, the highest and lowest temperatures on the midline of the induction pan are settling around at $270{ }^{\circ} \mathrm{C}$ and $180{ }^{\circ} \mathrm{C}$ respectively.

The thermal field computation results shown in Fig. 5 and Fig. 6 prove that the proposed square 9-coil system can achieve significant improvement in heating performance in terms of both uniformity and heating efficiency. With regard to the exciting modes for the proposed system, it can be selected by considering the balance of uniformity performance and heating efficiency. The $120^{\circ}$ phase shifted exciting mode can realize higher heating efficiency at the expense of less uniformity. Only simple exciting modes have been studied. It is however possible to implement more sophisticated excitation profiles, involving varying phase difference, or based on entirely different approach, so that the heating performance can be further optimised.

Nevertheless, even with simple excitation schemes on the novel 9-coil system, the above results show significant improvements in heating performance over traditional single coil systems.

\section{Conclusions}

This paper has proposed a novel square 9-coil system for induction cooker applications for improving the heating performance. On the basis of FEM analysis, three different exciting modes for the proposed system are thoroughly investigated. The magnetic field and eddy currents distribution are studied for each case. Two excitation modes are selected for further study on the thermal field computation in order to analyze their heating performance. The thermal field computation results indicate that the proposed square 9coil system can effectively enhance the heating performance in terms of uniformity and heating efficiency, using relatively simple excitation schemes. The simulation results provide insightful information and understanding for the design and optimization of the induction cooker with a square 9-coil system. Further work will focus on experimental validation of the proposed system, as well as more sophisticated excitation scheme to further enhance the heating performance.

\section{Acknowledgements}

The research is supported by the Research Grant Council (RGC) of Hong Kong under the grand PolyU 5133/08E, and the joint collaboration between Hong Kong PolyU and Cranfield University is promoted by the MOU within the Power Electronics Consortium, of which the two universities are a key member.

\section{References}

[1] F. Forest, S. Faucher, J.Y. Gaspard, D. Montloup, J.J. Huselstein, and C. Joubert, "Frequency-synchronized resonant converters for the supply of multiwinding coils in induction cooking appliances", IEEE Trans. Ind. Electron., 51, pp. 441-452, (2007).

[2] H. Kagimoto, D. Miyagi, N. Takahashi, N. Uchida, and K. Kawanaka, "Effect of temperature dependence of magnetic properties on heating characteristics of induction heater", IEEE Trans. Magn., 46, pp. 30183021, (2010).

[3] L.C. Meng, K.W.E. Cheng, and W.M. Wang, "Thermal impacts of electromagnetic proximity effects in induction cooking system with distributed planar multicoils", IEEE Trans. Magn., 47, pp. 3212-3215, (2011). 